\title{
Determinação da capacidade antioxidante de produtos naturais in vitro pelo método do DPPH॰: estudo de revisão
}

\section{OLIVEIRA, G.L.S.}

Curso de Licenciatura em Ciências Biológicas, Instituto Federal de Educação, Ciência e Tecnologia do Piauí, Praça da Liberdade, 1597, 64000-040, Centro, Teresina (PI) - Brasil. georgenota10@hotmail.com

\begin{abstract}
RESUMO: Os antioxidantes podem ser de grande benefício para a melhoria da qualidade de vida, já que eles têm a capacidade de proteger um organismo dos danos causados pelos radicais livres, prevenindo ou adiando o início de várias doenças. Uma das técnicas atualmente utilizada para detectar a presença de compostos antioxidantes, é um método baseado na eliminação do radical livre estável 1,1-difenil-2-picrilhidrazil (DPPH•). Este método é considerado fácil, preciso, rápido, simples, e econômico, sendo adequado para a determinação da capacidade antioxidante de substâncias puras e misturas. O objetivo deste artigo de revisão é fornecer informações sobre esse método. A pesquisa foi realizada usando o termo radical DPPH•, capacidade antioxidante-DPPH• e método DPPH• em periódicos, tais como: Pubmed, Wiley Online Library, ACS Publications, SpringerLink e ScinceDirect até janeiro de 2014.
\end{abstract}

Palavras-chave: Antioxidantes, método DPPH•, capacidade antioxidante.

\begin{abstract}
Determination in vitro of the antioxidant capacity of natural products by the DPPH॰method: review study. Antioxidants can be of great benefit to improve the quality of life, since they have the ability to protect an organism from damage caused by free radicals, preventing or delaying the onset of various diseases. One of the techniques currently used to detect the presence of antioxidant compounds is a method based on the elimination of the stable free radical 1,1-diphenyl-2-picrylhydrazyl (DPPH•). This method is considered easy, accurate, rapid, simple and inexpensive, and it is suitable for the determination of the antioxidant capacity of pure substances and blends. The aim of this review article is to provide information on this method. This research was performed using the terms DPPH • radical, antioxidant capacity $\mathrm{DPPH} \cdot$ and DPPH$\bullet$ method in journals such as Pubmed, Wiley Online Library, ACS Publications, SpringerLink and ScinceDirect up to January 2014.
\end{abstract}

Key words: Antioxidants, DPPH• method, antioxidant capacity.

\section{MÉTODO DO DPPH•}

Um amplo acervo de métodos está disponível na literatura científica para mensurar a capacidade antioxidante de vários tipos de substâncias. $O$ interesse em avaliar a capacidade antioxidante é resultado de vários estudos sobre a importância dos antioxidantes em sistemas biológicos (Karadag; Ozcelik; Saner, 2009; Pérezjiménez et al., 2008). Os antioxidantes podem ser de grande benefício para a melhoria da qualidade de vida, já que eles têm a capacidade de proteger um organismo dos danos causados pelos radicais livres, prevenindo ou adiando o início de várias doenças, como cardiovasculares, crônicas (câncer, aterosclerose, artrite reumática, hipertrofia muscular) e neurodegenerativas (Mal de Alzheimer) (Alam; Bristi; Rafiquzzaman, 2012; Halliwell e Gutteridge,
2007; Tinkel; Hassanain; Khouri, 2012; Borut e Raja, 2012).

Há um crescente interesse de pesquisadores no desenvolvimento de substâncias antioxidantes, principalmente a partir de produtos naturais como plantas. Assim, uma das técnicas atualmente utilizada para detectar a capacidade antioxidantes de compostos, é o método baseado na eliminação do radical livre estável 1,1-difenil-2-picrilhidrazil $(\mathrm{DPPH} \bullet)$ (Figura 1). A molécula de DPPH• é bastante conhecida por caracteriza-se como um radical orgânico livre estável e tem muitas vantagens, tais como uma boa estabilidade na ausência da luz, aplicabilidade, simplicidade e viabilidade (Deng; Cheng; Yang, 2011; Scherer; Godoy, 2009). Segundo Moon; Shibamoto (2009), o método 
DPPH• é utilizado em mais de $90 \%$ dos estudos de avaliação antioxidante de substâncias puras, misturas ou matrizes complexas.

O método DPPH• é bastante utilizado para avaliação da capacidade antioxidante, mas essa avaliação antioxidante não deve se basear apenas em uma única metodologia, sendo necessários outros métodos para caracterizar completamente um composto como antioxidante, como o método

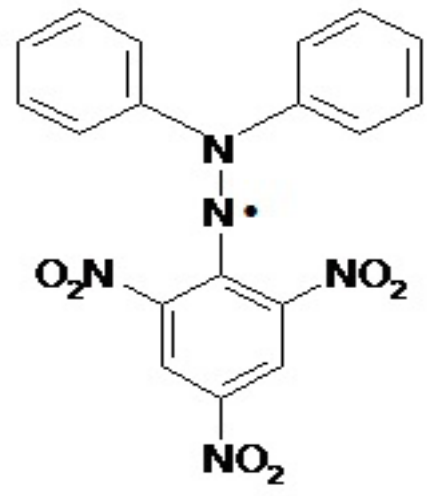

FIGURA 1. Estrutura do radical livre estável DPPH•

TEAC (Trolox Equivalent Antioxidant Capacity) ou ABTS •+ (ácido 2,2'-azinobis-3-etilbenzotiazolina6-sulfónico), ORAC (Oxygen radical absorbance capacity), FRAP (Ferric-Reducing Ability of Plasma), TRAP (Total Radical - Trapping Antioxidant Parameter), CUPRAC (Cupric ions $\mathrm{Cu}^{2+}$ reducing antioxidant power) e métodos de peroxidação lipídica (Ciesla et al., 2012; Moon; Shibamoto, 2009). A maioria das metodologias antioxidantes emprega basicamente o mesmo princípio, onde um radical sintético é gerado e a capacidade de uma amostra para eliminar ou neutralizar o radical é monitorada através de um espectrofotômetro UV/visível (Arnao, 2000).

Existem dois tipos de mecanismos de reação que acontecem na determinação da capacidade antioxidante, ambos resultando de neutralização ou redução de um radical. Um desses mecanismos se baseia na transferência de elétrons (TE) e o outro na transferência de um átomo de hidrogênio (TAH) (Gülçin, 2012; Huang; Prior, 2005; Karadag et al., 2009; Amatatongchai et al., 2012). Ambos os mecanismo de transferência de hidrogênio e elétrons para o radical DPPH• podem ser vistos na equação 1 e 2, respectivamente;

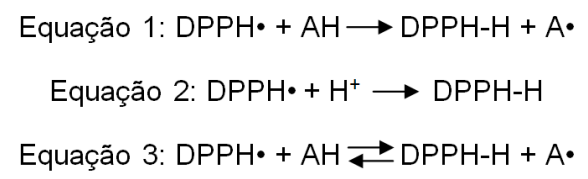

Para Karadag et al (2009), é difícil diferenciar esses dois mecanismos de reação para o método DPPH• e segundo Huang et al. (2005), o principal mecanismo para o método DPPH • acontece pela transferência de elétron e que a transferência de hidrogénio é uma via de reação marginal (Figura 2). Além disso, o mecanismo de reação de transferência de hidrogênio entre o radical DPPH• e o antioxidante $(\mathrm{AH})$ pode ser reversível, sendo que o $\mathrm{DPPH}-\mathrm{H}$ poderá se converter na forma de DPPH• (Bondet; Brand-Williams; Berset, 1997; Foti; Daquino, 2006). Esse mecanismo pode ser observado na equação 3.

O método DPPH• é considerado fácil, altamente sensível, preciso, rápido, simples, econômico e o radical DPPH• não precisa ser gerada e o sistema de reação envolve somente o radical e o antioxidante (Figura 2) (Kedare; Singh, 2011; Moon; Shibamoto, 2009). O método DPPH• foi desenvolvido por Blois (1958) para determinar a atividade antioxidante de varias substâncias utilizando um radical livre estável semelhante, $\alpha$-difenil- $\beta$-picrilhidrazila. Esse método foi depois modificado por Brand-Williams; Cuvelier; Berset (1995) para determinar o potencial antioxidante de compostos fenólicos e de amostras biológicas, simplificando a interpretação do resultado realizado
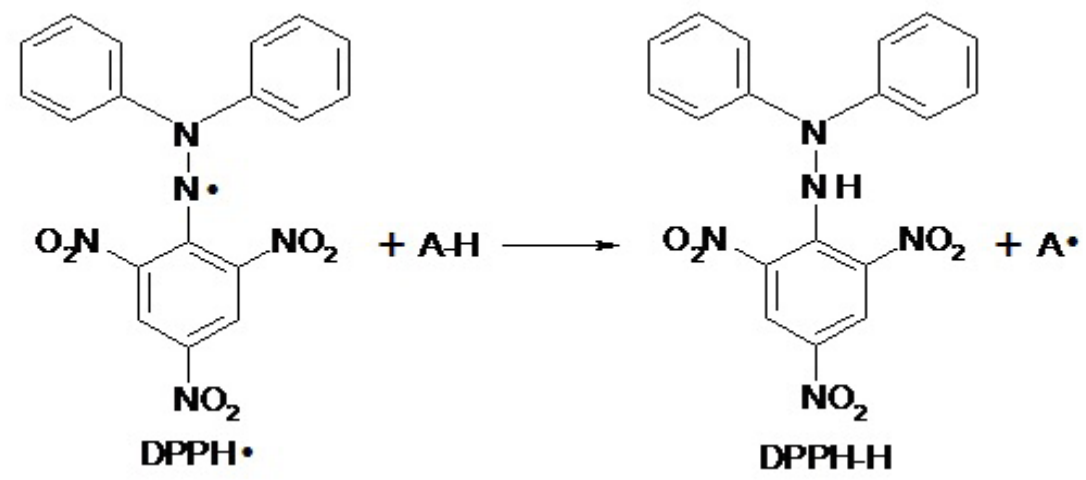

FIGURA 2. Mecanismos de reação entre o radical DPPH•e um antioxidante através da transferência de um átomo de hidrogênio. 
por Blois (1958) e utilizando o termo concentração eficaz que inibi $50 \%$ da concentração inicial do radical $\mathrm{DPPH} \bullet\left(\mathrm{CE}_{50}\right)$ para a interpretação dos resultados do método de DPPH $\bullet$.

Esse método continua sofrendo muitas modificações ou adaptações e por isso vários procedimentos ou protocolos para o DPPH• têm sido relatados, incluindo diferentes solventes para dissolver o radical DPPH • diferentes concentrações iniciais da solução de DPPH•, diferentes alíquotas das várias substâncias utilizadas e da solução inicial de DPPH•, diferentes tempos de reação com o radical DPPH• e diferentes absorbâncias também têm sido relatadas (Tabela 1).

Os radicais livres contém elétron desemparelhado, sendo instáveis e bastantes reativos, mas o DPPH•, devido a sua estrutura química é um radical livre estável, pois possui três anéis aromáticos apresentando efeito de ressonância que é importante para estabilizar a carga eletrônica do radical DPPH•. A estabilização do radical DPPH• é atribuído também ao deslocamento do elétron desemparelhado sobre o radical $\mathrm{DPPH} \bullet$, nos três grupos de $\mathrm{NO}_{2}$ e nos dois átomos de nitrogênio, que são grupos que permitem o deslocamento de elétrons (FIGURA 3) (Martinez et al., 2006).

Por causa da localização do elétron livre ao longo da molécula de DPPH• (Figura 1 e 3 ), o radical possui à cor púrpura ou violeta com uma absorção em solução de etanol ou metanol a 515-520 nm (Deng et al., 2011). Uma substância antioxidante pode doar um átomo de hidrogênio ou transferir

TABELA 1. Modificações ou adaptações do método DPPH•

\begin{tabular}{|c|c|c|c|c|c|}
\hline & & étodo DPPH• & & & \\
\hline $\begin{array}{c}\text { Concentração } \\
\text { inicial da solução } \\
\text { de DPPH• }\end{array}$ & $\begin{array}{l}\text { Tempo de Reação } \\
\text { (minutos) }\end{array}$ & $\begin{array}{c}\text { Quantidade } \\
\text { da solução de } \\
\text { DPPH• utilizado }\end{array}$ & $\begin{array}{c}\text { Tipos de solventes } \\
\text { utilizados }\end{array}$ & Absorbância & Referência \\
\hline $0,1 \mathrm{mM}$ & 30 & $50 \mathrm{uL}$ & Metanol & $515 \mathrm{~nm}$ & (Yao et al., 2012) \\
\hline $0,5 \mathrm{mM}$ & 30 & $2 \mathrm{~mL}$ & Metanol & $517 \mathrm{~nm}$ & (Alam et al., 2012) \\
\hline $6 \times 10^{-5} \mathrm{M}$ & 1 e 5 & $975 \mathrm{uL}$ & Etanol & $515 \mathrm{~nm}$ & (Amira et al., 2012) \\
\hline $31,5 \mathrm{uM}$ & 0 a 120 & $1 \mathrm{~mL}$ & Etanol & $518 \mathrm{~nm}$ & (Fagali; Catalá, 2008) \\
\hline $0,06 \mathrm{mM}$ & 0 a 9 & $3,9 \mathrm{~mL}$ & Metanol & $515 \mathrm{~nm}$ & (Rufino et al., 2009) \\
\hline $3,8 \mathrm{mM}$ & 6 & $20 \mathrm{uL}$ & Etanol & $520 \mathrm{~nm}$ & (Moț; Silaghi-Dumitrescu; \\
\hline $100 \mathrm{mM}$ & 20 & $700 \mathrm{uL}$ & Metanol & $515 \mathrm{~nm}$ & $\begin{array}{c}\text { Sârbu, 2011) } \\
\text { (Locatelli et al., 2009) }\end{array}$ \\
\hline $60 \mathrm{uM}$ & $\begin{array}{c}\text { Monitorado a até o estado } \\
\text { de equilíbrio ( } 6 \text { horas) }\end{array}$ & $1,95 \mathrm{~mL}$ & Metanol & $515 \mathrm{~nm}$ & (López-Giraldo et al., 2009) \\
\hline $0.4 \mathrm{mM} / \mathrm{L}$ & $\begin{array}{c}\text { Monitorado no tempo de } 30 \\
\text { minutos, } 2,6,12,24,36, \\
48,72,96,120 \text { e } 144 \text { horas }\end{array}$ & $0,5 \mathrm{~mL}$ & Etanol & 517 & (Liang et al., 2010) \\
\hline $100 \mathrm{mM}$ & Monitorado ao longo de $5 \mathrm{~h}$ & $2,9 \mathrm{~mL}$ & Etanol & 516 & (Ordoudi et al., 2006) \\
\hline $0,3 \mathrm{mM}$ & $\begin{array}{c}\text { A cada } 10 \text { minutos até } 1 \\
\text { hora }\end{array}$ & $1 \mathrm{~mL}$ & Etanol & 540 & (Liu et al., 2008) \\
\hline $0,1 \mathrm{mM}$ & $\begin{array}{c}\text { A cada } 15 \text { minutos até o } \\
\text { estado de equilíbrio }\end{array}$ & $2 \mathrm{~mL}$ & Metanol & 517 & (Lu; Yeap Foo, 2000) \\
\hline $100 \mu \mathrm{M}$ & 30 & 2,95 & Acetato de etila & 520 & (Prevc et al., 2013) \\
\hline $0.025 \mathrm{mM}$ & 180 & $20 \mathrm{~mL}$ & $\begin{array}{l}\text { Tampão fosfato de } \\
\text { sódio em pH } 7.0\end{array}$ & 517 & (Niu et al., 2012) \\
\hline $1.55 \times 10^{-4} \mathrm{M}$ & 60 & - & $\begin{array}{l}\text { Agua e etanol em } \\
\text { uma mistura de } 1 / 1\end{array}$ & 524 & (Friaa et al., 2008) \\
\hline $3.8 \mathrm{mg} / 50 \mathrm{~mL}$ & 30 & $0,975 \mathrm{~mL}$ & $\begin{array}{c}\text { Acetona, hexano e } \\
\text { metanol }\end{array}$ & 515 & $\begin{array}{c}\text { (Fernandez-Orozco et al., } \\
\text { 2011) }\end{array}$ \\
\hline $0,4 \mathrm{mM}$ & 30 & $3 \mathrm{~mL}$ & $\begin{array}{c}\text { Solução metanólica } \\
0,004 \%\end{array}$ & 517 & (Liang; Jin; Liu, 2011) \\
\hline $0.05 \mathrm{mM}$ & 5 & $0,5 \mathrm{~mL}$ & $\begin{array}{c}\text { Solução aquosa de } \\
\text { micela }\end{array}$ & 528 & (Noipa et al., 2011) \\
\hline
\end{tabular}

Rev. Bras. PI. Med., Campinas, v.17, n.1, p.36-44, 2015. 


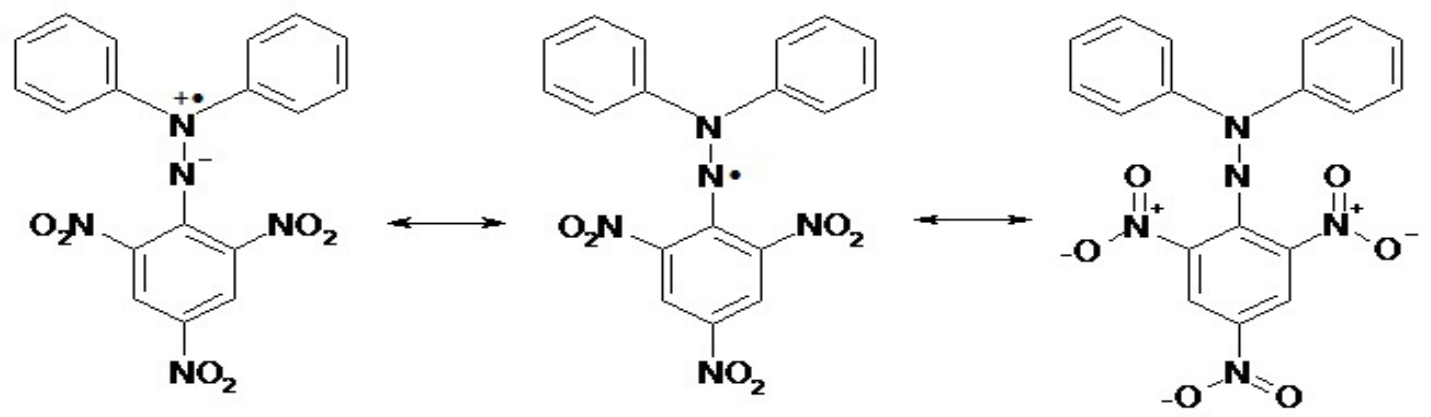

FIGURA 3. Estabilização do radical DPPH• pelo deslocamento do elétron desemparelhado.

um elétron para a molécula de DPPH•, que aceita para se tornar uma molécula estável diamagnético originando a forma reduzida DPPH-H com a perda da cor violeta de acordo com o tempo para amarelo pálido ou violeta claro (Cieśla et al., 2012; Kedare; Singh, 2011; Amatatongchai et al., 2012; Musa et al., 2013). A mudança da cor violeta escuro para violeta claro, que resulta na diminuição da absorbância do radical DPPH•, pode ser monitorada por um espectrofotômetro UV/visível para a determinação capacidade antioxidante. Esse monitoramento tem que ser realizado sempre no escuro, por que a luz é um fator que interfere diretamente na reação do radical DPPH • com uma substância, acelerando a diminuição da absorbância e consequente alterando os resultados finais (Scherer; Godoy, 2009; Mishra; Ojha; Chaudhury, 2012).

Vários métodos de avaliação antioxidante in vitro e in vivo em amostras (extratos de plantas, alimentos e antioxidantes sintéticos) são utilizados pela comunidade cientifica. Dentre os métodos de avaliação antioxidante in vitro, o DPPH• é bastante utilizado e por isso, o objetivo deste artigo de revisão é fornecer informações desse método como princípios químicos, limitações, desvantagens e interpretação dos resultados. Esta revisão não será tão abrangente, já que apenas alguns tópicos importantes sobre o método DPPH• serão abordados, de modo que muitos artigos não serão citados. A pesquisa foi realizada usando o termo radical $\mathrm{DPPH} \bullet$, capacidade antioxidante-DPPH $\bullet \mathrm{e}$ método DPPH• em periódicos como o da Pubmed, Wiley Online Library, ACS Publications, SpringerLink e ScinceDirect até janeiro de 2014.

\section{Limitações e desvantagens da aplicação do método DPPH para avaliar a capacidade antioxidante}

Desde a primeira aplicação até os dias atuais, o método DPPH• sofreu muitas modificações e consequentemente diferentes protocolos tem sido usado, tornando complicado fazer comparações com resultados de avaliação antioxidante por diferentes protocolos. Durante o desenvolvimento desta pesquisa nos periódicos da Pubmed, Wiley Online Library, ACS Publications, SpringerLink e ScinceDirect, os artigos que abordavam o método DPPH - utilizavam procedimentos bastantes diferentes (Tabela 1). Levando em consideração os diferentes protocolos desenvolvidos, confirma-se a necessidade de se padronizar o método DPPH $\bullet$. Autores como Cieśla et al. (2012), Cheng; Moore; Yu (2006); Chandrasekar et al. (2006), Polášek et al. (2004), Sharma; Bhat (2009), Deng et al. (2011), Locatelli et al. (2009), Noipa et al. (2011) e Scherer; Godoy (2009) buscaram desenvolver procedimentos com o objetivo de padronização para avaliação da capacidade antioxidante pelo método DPPH .

A padronização do método DPPH• elimina umas das principais desvantagens desse método, que seria as diferentes maneiras de interpretar e determinar a capacidade antioxidante de uma determinada substância. Nem sempre o mesmo método utiliza o mesmo procedimento e como consequência os diferentes resultados obtidos em varias pesquisas não podem ser comparados (Cheng; Moore; Yu 2006; Alam; Bristi; Rafiquzzaman, 2012; Pérez-jiménez et al., 2008). Segundo os trabalhos de Pérez-Jiménez; Saura-Calixto (2006), os resultados obtidos da capacidade antioxidante só deve ser comparada quando as medições foram feitas pelo mesmo procedimento, como a utilização do mesmo tipo de solvente.

Apesar de o método DPPH • ser fácil e simples de realizar existe alguns inconvenientes que limitam a sua aplicação, como por exemplo, o tipo de solvente utilizado para dissolver o radical $\mathrm{DPPH} \cdot$, que tem maior solubilidade em meios orgânicos como etanol e metanol (Arnao, 2000). Em meios alcoólicos, há uma maior facilidade da doação de um átomo de hidrogênio do próprio álcool (etanol ou metanol), que aumenta a solubilidade e a taxa constante de transferência de hidrogênio para o radical DPPH•, principalmente para o átomo de nitrogênio que está ligado aos dois anéis aromáticos. Assim, a carga eletrônica que antes estava se deslocando sobre o radical DPPH• (Figura 3 ), se restringe mais ao átomo de nitrogênio $\left(\mathrm{N}_{\mathrm{a}}\right)$ localizado no meio do 
radical $\mathrm{DPPH} \cdot$, aumentando a reatividade do radical com uma substância antioxidante (AH) (Figura 4) (Tsimogiannis; Oreopoulou, 2004; Valgimigli et al., 1995).

Em meios aquosos a solubilidade é bem menor e pode interferir na avaliação da capacidade antioxidante, tornando o radical DPPH• pouco acessível para a reação com as amostras antioxidantes, afetando assim a transferência de elétron ou de hidrogênio (Karadag et al., 2009; Magalhães et al., 2008). Consequentemente, a cinética de reação do radical DPPH $\operatorname{com} o$ antioxidante depende bastante do tipo de solvente, sendo que a influência do tipo de solvente é geralmente omitida em vários procedimentos de avaliação antioxidante (Dawidowicz; Wianowska; Olszowy, 2012). Noipa et al. (2011) desenvolveu um método DPPH• em solução aquosa usando agregados de surfactante ou sistemas de micelas, demonstrando que esse método pode ser usado para determinar a capacidade antioxidante tanto em meio orgânico quanto em meio aquoso.

Além do inconveniente do tipo de solvente, o método DPPH• sofre bastante influência da luz e do $\mathrm{pH}$ do solvente utilizado para dissolver o radical DPPH•, sendo que todo o procedimento de avaliação antioxidante tem que ser realizado no escuro (Magalhães et al., 2008; Gülçin, 2012; Cieśla et al., 2012). No trabalho desenvolvido por Ozcelik; Lee; Min (2003), foi observado que há uma perda da absorbância da reação entre a solução de $\mathrm{DPPH} \bullet$ e o antioxidante, em torno de 20 a 35\% na presença de luz. Essa perda ainda pode ser maior de acordo com o tempo. A presença de compostos não antioxidantes nas soluções testadas podem também interferir na avaliação antioxidante, alterando principalmente os resultados obtidos (Karadag et al., 2009; Kedare; Singh, 2011; Pérez-jiménez et al., 2008; Dawidowicz; Wianowska; Olszowy, 2012).

Como dito anteriormente, o DPPH• é um radical livre estável e não apresenta semelhança com os radicais livres mais reativos e não pode ser correlacionados a sistemas biológicos, ou seja, não é fisiologicamente relevante. (Huang et al., 2005;
Prior; Wu; Schaich, 2005). O método DPPH• não é apropriado para medir a capacidade antioxidante de plasma e soro humano, porque as proteínas podem precipitar no meio da reação em solução alcoólica (Karadag et al., 2009). No entanto, o método $\mathrm{DPPH} \cdot$ tem aplicação em soro desproteinado, sendo o estudo antioxidante em soro humano uma ferramenta importante (Chrzczanowicz et al., 2008; Magalhães et al., 2008; Martinez et al., 2006; Rysz et al., 2010).

Interpretação dos resultados experimentais da capacidade antioxidante pelo método DPPH.

Os resultados obtidos da capacidade antioxidante pelo método DPPH - têm sido apresentados de diversas formas e a falta de padronização dos resultados torna difícil comparar a capacidade antioxidante de uma mesma amostra, ou diferentes amostras (Deng et al., 2011). A maioria dos resultados é apresentada como valor de $\mathrm{CE}_{50} \mathrm{Ou} \mathrm{Cl} \mathrm{Cl}_{50}$, que é definida como a quantidade de antioxidante necessário para diminuir ou reduzir a concentração inicial do radical DPPH• em 50\% (Brand-Williams et al., 1995; Chen; Bertin; Froldi, 2012). Este valor é calculado através da representação gráfica da inibição percentual do radical DPPH• em função da concentração do antioxidante (Brand-Williams et al., 1995). Apesar dos resultados antioxidante ser expresso como $\mathrm{CE}_{50}$, os estudos experimentais realizados por Eklund et al. (2005) e Villano et al. (2005) indicaram uma relação não linear entre a concentração do antioxidante e a atividade sequestradora do radical $\mathrm{DPPH} \cdot$, e por isso, a interpretação dos resultados antioxidante pelo valor da $\mathrm{CE}_{50}$ pode não ser adequado. Levando em consideração esse problema, Locatelli et al. (2009) desenvolveu um método para calcular com precisão o valor da $\mathrm{CE}_{50}$ de substâncias que inibi o radical livre $\mathrm{DPPH} \cdot$. Um software matemático foi desenvolvido para interpretar os dados e obter uma relação entre a atividade antioxidante e a concentração da amostra. Três diferentes modelos de regressão (Probit, Logit e Angular) foram consideradas para o cálculo

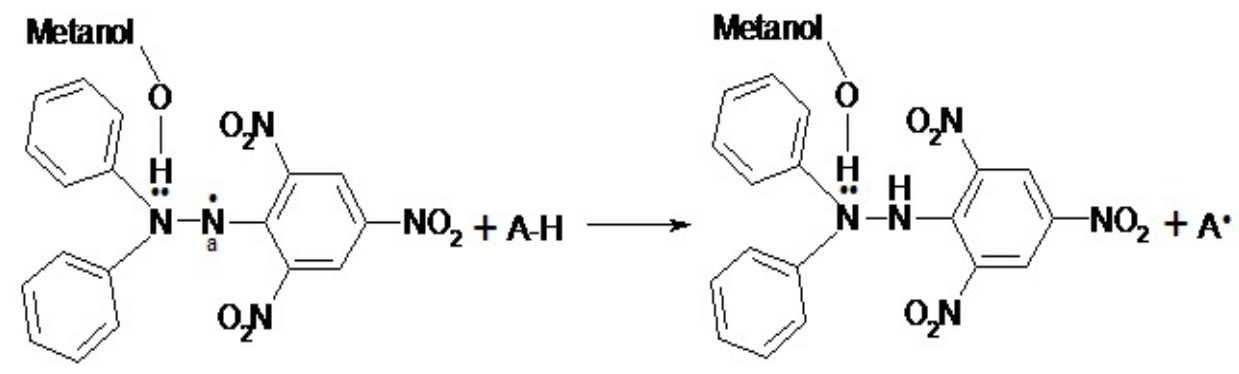

FIGURA 4. Ligação química do metanol com o radical DPPH• e o aumento da localização do elétron desemparelhado no átomo de nitrogênio $\left(\mathrm{N}_{\mathrm{a}}\right)$ do radical DPPH $\bullet$. 
dos valores do $C_{50}$. A regressão probit foi a mais eficaz para calcular os valores da $\mathrm{CE}_{50}$ de extratos naturais, alimentos e de compostos como ácido gálico e quercetina, mostrando altos coeficientes de correlação. Para Chen; Bertin e Froldi (2013), esse problema também poderia ser solucionado por meio de uma equação matemática adequada e por isso, em seu estudo, a $\mathrm{CE}_{50}$ da quercetina, catequina, ácido ascórbico, ácido caféico, ácido clorogênico e acetilcisteína foram determinados utilizando uma abordagem comparativa entre vários modelos de regressão nos programas de estatísticas GraphPad Prism $^{\circledast}$ 5.01, BLeSq, OriginPro ${ }^{\circledR}$ 8.5.1, SigmaPlot ${ }^{\circledR} 12$, BioDataFit 1.02 e IBM SPSS Statistics ${ }^{\circledR}$ Desktop 19.0., em que foi observado que na análise dos parâmetros de regressão, o GraphPad Prism ${ }^{\circledR}$ apresentou um melhor desempenho e uma menor variação em relação ao valor real da $\mathrm{CE}_{50}$.

Scherer; Godoy (2009) consideram que não existe uma avaliação da capacidade antioxidante universal para o método DPPH • e por isso desenvolveu um procedimento padrão através da fórmula que expressa os resultados da capacidade antioxidante como "Índice de atividade antioxidante(IAA)", que pode ser calculada pela seguinte fórmula:

$\mathrm{IAA}=$ Concentração final do DPPH $(\mu \mathrm{g} / \mathrm{mL}) / \mathrm{CE}_{50}(\mu \mathrm{g} / \mathrm{mL})$

Scherer; Godoy (2009) consideraram que as amostras em análise apresentam atividade antioxidante pobre quando IAA for menor que 0,5, atividade antioxidante moderada quando IAA estiver entre 0,5 e 1,0, atividade antioxidante forte quando IAA estiver entre 1,0 e 2,0, e atividade antioxidante muito forte quando IAA for maior que 2,0. O IAA mostrou-se adequado para a interpretação dos resultados da capacidade antioxidante de extratos de plantas e de compostos puros, sendo que não houve diferença significativa no IAA quando diferentes soluções e concentrações do DPPH• e de várias substâncias foram utilizadas.

Deng et al. (2011) propôs a interpretação dos resultados da capacidade antioxidante de produtos naturais utilizando o método DPPH • como Unidade de Atividade Antioxidante (UAA). Sua definição é que um mol de radical DPPH• é completamente reduzida por um mol de antioxidante. Ele considera que UAA é adequado para comparar a capacidade antioxidante de extratos de plantas e de compostos puros, mesmo quando diferentes concentrações são usadas, o que torna este método muito prático e permite sua padronização.

Utilizando o método DPPH•, SánchezMoreno; Larrauri; Saura-Calixto (1998) classificaram a capacidade antioxidante de alguns compostos fenólicos de acordo com o comportamento cinético, baseado no valor de $\mathrm{T}_{\mathrm{CE} 50}$, como rápido quando $\mathrm{O}$ comportamento cinético for menor que 5 minutos, intermediário no intervalo entre 5 e 30 minutos, e lento quando é maior que 30 minutos. O tempo necessário para atingir o estado de equilíbrio com a $\mathrm{CE}_{50}$ é definido como $\mathrm{T}_{\mathrm{CE} 50^{\circ}}$. Levando em consideração o valor do $T_{C E 50}$, foi proposta uma fórmula para expressar a capacidade antioxidante de uma determinada substância, denominado de Eficiência Antiradical (EA), que pode ser calculado pela seguinte fórmula:

\section{$\mathrm{EA}=1 / \mathrm{CE}_{50} \mathrm{~T}_{\mathrm{CE} 50}$}

$O$ valor da EA foi utilizado para classificar a capacidade antioxidante em baixa (quando o valor do EA é menor que $1 \times 10^{-3}$ ), média (quando o valor do EA está entre $1 \times 10^{-3}$ e $5 \times 10^{-3}$ ), alta (quando o valor do EA está entre $5 \times 10^{-3}$ e $10 \times 10^{-3}$ ) e super alta (quando o valor do EA é maior que $10 \times 10^{-3}$ ). Uma das desvantagens dessa interpretação, é que o $\mathrm{AE}$ e o $T_{\text {CE5 } 0}$ não foram totalmente definidos, sendo que $O$ $\mathrm{T}_{\mathrm{CE} 50}$ foi determinadoa partir de um gráfico secundário que leva em consideração o tempo do estado estacionário e a concentração do antioxidante. Logo, pode existir uma grande variação nos valores de $T_{C E 50}$, já que não há uma relação linear entre o tempo no estado de equilíbrio e a concentração do antioxidante (Cheng et al., 2006).

Mishra; Ojha; Chaudhury (2012) utilizaram quase o mesmo parâmetro estabelecido por Sánchez-Moreno; Larrauri; Saura-Calixto (1998), mas não utilizou o valor da $T_{C E 50}$ e obteve os resultados antioxidantes como Antioxidant Reducing Power(ARP), que pode ser calculada pela seguinte formula:

$$
\mathrm{ARP}=1 / \mathrm{CE}_{50,}
$$

em que, ARP é definido como o inverso da $\mathrm{CE}_{50}$. Mishra; Ojha; Chaudhury (2012) também classificaram a capacidade antioxidante de determinados compostos considerando a cinética de reação do radical DPPH • com o antioxidante, nas categorias de rápido (tempo de reação menor que 30 minutos), médio (tempo de reação entre 30 minutos e uma 1 hora) e lento (tempo de reação maior que 1 hora).

A interpretação da absorbância de uma reação entre o radical $\mathrm{DPPH} \cdot \mathrm{e}$ um antioxidante pode ser determinado, de acordo com o tempo, como porcentagem de radical DPPH • remanescente, que pode ser calculado pela seguinte fórmula:

$\%$ de DPPH $\bullet_{\text {Remanescente }}=\left[(\mathrm{DPPH} \bullet)_{T=t} /(\mathrm{DPPH} \bullet)_{T=0}\right] \times 100$,

em que, $(\mathrm{DPPH} \cdot)_{T=t}$ é o valor da concentração do DPPH• após a reação com uma substâncias e $(\mathrm{DPPH} \bullet)_{T=0}$ é o valor da concentração inicial da solução de DPPH• no tempo de 0 minuto (Brand-

Rev. Bras. PI. Med., Campinas, v.17, n.1, p.36-44, 2015. 
Williams et al., 1995; Dawidowicz et al., 2012; Huang et al., 2005; Karadag et al., 2009; SánchezMoreno; Larrauri; Saura-Calixto, 1998). Cheng et al. (2006) obteve a porcentagem de radical DPPH• remanescente, no tempo de 40 minutos, de acordo com a seguinte fórmula:

$\%$ de DPPH $\bullet_{\text {Remanescente }}=\left[\left(A_{\text {amostra }}-A_{\text {branco }}\right) /\left(A_{\text {controle }}-A_{\text {branco }}\right)\right] \times 100$,

em que, $A_{\text {amostra }}$ é o absorbância da reação entre a solução do radical DPPH• e a amostra antioxidante, $\mathrm{A}_{\text {branco }}$ é a absorbância da solução de solvente utilizado para preparar a amostra antioxidante, $\mathrm{A}_{\text {controle }}$ é a absorbância do radical DPPH• com uma pequena alíquota do solvente utilizado para preparar a amostra, em substituição à solução da própria amostra em estudo.

A monitoração da diminuição da absorbância da reação entre a solução de DPPH• e uma amostra pode ser determinada também como porcentagem de inibição, sequestro ou eliminação do radical $\mathrm{DPPH} \bullet$, que pode ser obtido pela fórmula:

$\%$ de inibição do radical DPPH $\bullet=\left[\left(\mathrm{A}_{\text {controle }}-\mathrm{A}_{\text {amostra }}\right) / \mathrm{A}_{\text {controle }}\right.$ $\mathrm{x} 100]$.

Cheng et al. (2006) estimou a porcentagem de inibição ou varrimento de radical $\mathrm{DPPH} \cdot$ de acordo com a seguinte fórmula;

\% de inibição do radical DPPH $\left[1-\left(A_{\text {amostra }}-A_{\text {branco }}\right) /\left(A_{\text {controle }}-\right.\right.$ $\left.\left.\mathrm{A}_{\text {branco }}\right)\right] \times 100$.

Dawidowicz et al. (2012) determinou a porcentagem de inibição do radical DPPH• através da seguinte fórmula;

$\%$ de inibição do radical DPPH• $=100-\% \mathrm{DPPH}_{\text {Remanescente }}$.

Trabalhando com várias mistura de compostos antioxidantes puros, Liu et al. (2008) expressou a capacidade antioxidante como Efeito Sinérgico (ES). O ES significa que as misturas de dois ou três compostos podem resultar numa maior capacidade antioxidante do que o apresentado por cada composto individualmente (Castro et al., 2006). Para o cálculo do ES das misturas antioxidantes, Liu et al. (2008) obteve primeiro o valor da capacidade de eliminação experimental (CEE), que pode ser determinado pela seguinte fórmula;

$$
\left.\% \text { CEE }=100-\left[\left(A_{\text {amostra }}-A_{\text {branco }}\right) \times 100\right) / A_{\text {controlo }}\right] \text {. }
$$

Depois de obter o valor do CEE, Liu et al. (2008) calculou o valor da capacidade de eliminação teórica (CET), que é a soma do valor CEE (CEE $\mathrm{CEE}_{2}, \mathrm{CEE}_{3}, \mathrm{CEE}_{4}$ ) referente a cada composto antioxidante, pela seguinte fórmula;

$\%$ CET $=100-\left[\left(100-\mathrm{CEE}_{1}\right) \times\left(100-\mathrm{CEE}_{2} / 100\right) \times(100\right.$

$\left.\left.-\mathrm{CEE}_{3} / 100\right) \times\left(100-\mathrm{CEE}_{4} / 100\right)\right]$

Logo, o valor do ES de uma mistura de antioxidante pode ser determinado pela seguinte formula;

$E S=C E E / C E T$,

onde, uma mistura antioxidante apresenta efeito sinérgico se o valor do ES for maior que 1. Vale ressaltar que a capacidade antioxidante expressa como ES, é utilizado somente para misturas de compostos puros e por isso não é utilizado para extratos de plantas.

O valor da absorbância pode ser ainda interpolado em uma curva de dose-resposta de um antioxidante padrão, como ácido ascórbico ou Trolox, ácido tânico e ácido gálico e os resultados são expressos como concentração equivalente (Kedare; Singh, 2011; Magalhães et al., 2008; Arnao, 2000).

Soler-Rivas; Espín; Wichers (2000) sugeriu uma versão mais rápida e simples do método DPPH• em cromatografia de camada delgada (CCD). O surgimento de um halo branco ou manchas amareladas no fundo roxo ao redor da amostra colocada na CCD, quando se aplica o radical $\mathrm{DPPH} \cdot$ sobre o CCD, se torna um indicativo da capacidade antioxidante. Cieśla et al. (2012) buscou desenvolver um procedimento padrão para o DPPH• em CCD, levando em consideração a influência do tipo de adsorvente, o tempo entre a imersão e a reação e o solvente utilizado para dissolução do $\mathrm{DPPH} \bullet$. Os resultados obtidos desse procedimento foram interpretados de forma qualitativa através de imagens e videoscans, obtidos por meio de um programa de processamento de imagem.

\section{REFERÊNCIAS}

ALAM, M. N.; BRISTI, N. J.; RAFIQUZZAMAN, M. Review on in vivo and in vitro methods evaluation of antioxidant activity. Saudi Pharmaceutical Journal, v. 21, n. 2, p. 143-152, 2013.

AMATATONGCHAI, M. et al. Simple flow injection for screening of total antioxidant capacity by amperometric detection of DPPH radical on carbon nanotube modifiedglassy carbon electrode. Talanta, v. 97, n. 0, p. 267-272, 2012.

AMIRA, E. A. et al. Effects of the Ripening Stage on Phenolic Profile, Phytochemical Composition and Antioxidant Activity of Date Palm Fruit. Journal of Agricultural and Food Chemistry, 2012.

ARNAO, M. B. Some methodological problems in the 
determination of antioxidant activity using chromogen radicals: a practical case. Trends in Food Science \&amp; Technology, v. 11, n. 11, p. 419-421, 2000.

BLOIS, M. S. Antioxidant Determinations by the Use of a Stable Free Radical. Nature, v. 181, n. 4617, p. 11991200, 1958

BONDET, V.; BRAND-WILLIAMS, W.; BERSET, C. Kinetics and Mechanisms of Antioxidant Activity using the DPPH.Free Radical Method. LWT - Food Science and Technology, v. 30, n. 6, p. 609-615, 1997.

BORUT, P.; RAJA, D. Free Radicals and Extrinsic Skin Aging. Dermatology Research and Practice, v. 2012, p. 1-4, 2012.

BRAND-WILLIAMS, W.; CUVELIER, M. E.; BERSET, C. Use of a free radical method to evaluate antioxidant activity. LWT - Food Science and Technology, v. 28, n. 1, p. 25-30, 1995.

CASTRO, I. A. et al. 2,2 -Diphenyl-1-picrylhydrazil free radical scavenging activity of antioxidant mixtures evaluated by response surface methodology. International Journal of Food Science \& Technology, v. 41, p. 59-67, 2006.

CHANDRASEKAR, D. et al. Determination of DPPH free radical scavenging activity by reversed-phase HPLC: a sensitive screening method for polyherbal formulations. J Pharm Biomed Anal, v. 40, n. 2, p. 460-4, 2006.

CHENG, Z.; MOORE, J.; YU, L. High-Throughput Relative DPPH Radical Scavenging Capacity Assay. Journal of Agricultural and Food Chemistry, v. 54, n. 20, p. 7429-7436, 2006.

CHRZCZANOWICZ, J. et al. Simple method for determining human serum 2,2-diphenyl-1-picrylhydrazyl (DPPH) radical scavenging activity - possible application in clinical studies on dietary antioxidants. Clin Chem Lab Med, v. 46, n. 3, p. 342-9, 2008.

CHEN, Z.; BERTIN, R.; FROLDI, G. EC E $_{50}$ estimation of antioxidant activity in DPPH assay using several statistical programs. Food Chemistry, v. 138, n. 1, p. 414-420, 2013.

CIEŚLA, Ł. et al. Approach to develop a standardized TLC-DPPH test for assessing free radical scavenging properties of selected phenolic compounds. Journal of Pharmaceutical and Biomedical Analysis, v. 70, n. 0, p. 126-135, 2012.

DAWIDOWICZ, A. L.; WIANOWSKA, D.; OLSZOWY, M. On practical problems in estimation of antioxidant activity of compounds by DPPH method (Problems in estimation of antioxidant activity). Food Chemistry, v. 131, n. 3, p. 1037-1043, 2012.

DENG, J.; CHENG, W.; YANG, G. A novel antioxidant activity index (AAU) for natural products using the DPPH assay. Food Chemistry, v. 125, n. 4, p. 1430-1435, 2011. ISSN 0308-8146.

EKLUND, P. C. et al. Chemical studies on antioxidant mechanisms and free radical scavenging properties of lignans. Organic \& Biomolecular Chemistry, v. 3, n. 18, p. 3336-3347, 2005.

FAGALI, N.; CATALÁ, A. Antioxidant activity of conjugated linoleic acid isomers, linoleic acid and its methyl ester determined by photoemission and DPPH techniques. Biophysical Chemistry, v. 137, n. 1, p. 56-62, 2008.

FERNANDEZ-OROZCO, R. et al. DPPH-scavenging capacity of chloroplastic pigments and phenolic compounds of olive fruits (cv. Arbequina) during ripening. Journal of Food Composition and Analysis, v. 24, n. 6, p. 858-864, 2011.

FOTI, M. C.; DAQUINO, C. Kinetic and thermodynamic parameters for the equilibrium reactions of phenols with the dpph[radical dot] radical. Chemical Communications, n. 30, p. 3252-3254, 2006.

FRIAA, O.; BRAULT, D. Kinetics of the reaction between the antioxidant Trolox and the free radical DPPH in semiaqueous solution. Organic \& Biomolecular Chemistry, v. 4 , n. 12, p. 2417-23, 2006.

GÜLÇIN, İ. Antioxidant activity of food constituents: an overview. Archives of Toxicology, v. 86, n. 3, p. 345391, 2012.

HUANG, D.; PRIOR, R. L. The Chemistry behind Antioxidant Capacity Assays. Journal of Agricultural and Food Chemistry, v. 53, n. 6, p. 1841-1856, 2005.

HALLIWELL, B.; GUTTERIDGE, J.M. C. Antioxidants: Molecules, medicines, and myths. Biochemical and Biophysical Research Communications, v. 393, p. 561564, 2010.

KARADAG, A.; OZCELIK, B.; SANER, S. Review of Methods to Determine Antioxidant Capacities. Food Analytical Methods, v. 2, n. 1, p. 41-60, 2009. ISSN 1936-9751.

KEDARE, S.; SINGH, R. Genesis and development of DPPH method of antioxidant assay. Journal of Food Science and Technology, v. 48, n. 4, p. 412-422, 2011.

KONDO, S. et al. Antioxidative activity of apple skin or flesh extracts associated with fruit development on selected apple cultivars. Scientia Horticulturae, v. 96 , n. 1-4, p. 177-185, 2002. ISSN 0304-4238.

LIANG, X.L. et al. Improved in Vitro Assays of Superoxide Anion and 1,1-Diphenyl- 2-picrylhydrazyl (DPPH) Radical-Scavenging Activity of Isoflavones and Isoflavone Metabolites. Journal of Agricultural and Food Chemistry, v. 58, n. 22, p. 11548-11552, 2010.

LIANG, B.; JIN, M.; LIU, H. Water-soluble polysaccharide from dried Lycium barbarum fruits: Isolation, structural features and antioxidant activity. Carbohydrate Polymers, v. 83, n. 4, p. 1947-1951, 2011.

LIU, D. et al. The scavenging capacity and synergistic effects of lycopene, vitamin $E$, vitamin $C$, and $\beta$-carotene mixtures on the DPPH free radical. LWT - Food Science and Technology, v. 41, n. 7, p. 1344-1349, 2008.

LOCATELLI, M. et al. Study of the DPPH-scavenging activity: Development of a free software for the correct interpretation of data. Food Chemistry, v. 114, n. 3, p. 889-897, 2009.

LÓPEZ-GIRALDO, L. J. et al. Kinetic and Stoichiometry of the Reaction of Chlorogenic Acid and Its Alkyl Esters against the DPPH Radical. Journal of Agricultural and Food Chemistry, v. 57, n. 3, p. 863-870, 2009.

LU, Y.; YEAP FOO, L. Antioxidant and radical scavenging activities of polyphenols from apple pomace. Food Chemistry, v. 68, n. 1, p. 81-85, 2000.

MAGALHÃES, L. M. et al. Methodological aspects about in vitro evaluation of antioxidant properties. Analytica Chimica Acta, v. 613, n. 1, p. 1-19, 2008.

MARTINEZ, S. et al. Cyclic voltammetry study of plasma antioxidant capacity - Comparison with the DPPH and TAS spectrophotometric methods. Journal of Electroanalytical Chemistry, v. 588, n. 1, p. 68-73, 2006.

Rev. Bras. PI. Med., Campinas, v.17, n.1, p.45-50, 2015. 
MISHRA, K.; OJHA, H.; CHAUDHURY, N. K. Estimation of antiradical properties of antioxidants using DPPH assay: A critical review and results. Food Chemistry, v. 130, n. 4, p. 1036-1043, 2012.

MOON, J.-K.; SHIBAMOTO, T. Antioxidant Assays for Plant and Food Components. J Agric Food Chem, v. 57, n. 5, p. 1655-1666, 2009.

MOT,, A. C.; SILAGHI-DUMITRESCU, R.; SÂRBU, C. Rapid and effective evaluation of the antioxidant capacity of propolis extracts using DPPH bleaching kinetic profiles, FT-IR and UV-vis spectroscopic data. Journal of Food Composition and Analysis, v. 24, n. 4-5, p. 516-522, 2011.

MUSA, K. H. et al. A novel high throughput method based on the DPPH dry reagent array for determination of antioxidant activity. Food Chemistry, v. 141, n. 4, p. 4102-4106, 2013.

NIU, Y. et al. Temperature-dependent stability and DPPH scavenging activity of liposomal curcumin at $\mathrm{pH}$ 7.0. Food Chemistry, v. 135, n. 3, p. 1377-1382, 2012.

NOIPA, T. et al. New approach for evaluation of the antioxidant capacity based on scavenging DPPH free radical in micelle systems. Food Research International, v. 44, n. 3, p. 798-806, 2011.

ORDOUDI, S. A. et al. Structure-DPPH• Scavenging Activity Relationships: Parallel Study of Catechol and Guaiacol Acid Derivatives. Journal of Agricultural and Food Chemistry, v. 54, n. 16, p. 5763-5768, 2006.

OZCELIK, B.; LEE, J. H.; MIN, D. B. Effects of Light, Oxygen, and $\mathrm{pH}$ on the Absorbance of 2,2-Diphenyl1-picrylhydrazyl. Journal of Food Science, v. 68, n. 2, p. 487-490, 2003.

PÉREZ-JIMÉNEZ, J. et al. Updated methodology to determine antioxidant capacity in plant foods, oils and beverages: Extraction, measurement and expression of results. Food Research International, v. 41, n. 3, p. 274-285, 2008

PÉREZ-JIMÉNEZ, J.; SAURA-CALIXTO, F. Effect of solvent and certain food constituents on different antioxidant capacity assays. Food Research International, v. 39, n. 7, p. 791-800, 2006.

POLÁŠEK, M. et al. Rapid automated assay of antioxidation/radical-scavenging activity of natural substances by sequential injection technique (SIA) using spectrophotometric detection. Analytical and Bioanalytical Chemistry, v. 379 , n. 5, p. 754-758, 2004.

PRIOR, R. L.; WU, X.; SCHAICH, K. Standardized Methods for the Determination of Antioxidant Capacity and Phenolics in Foods and Dietary Supplements. Journal of Agricultural and Food Chemistry, v. 53, n. 10 , p. $4290-4302,2005$.

PREVC, T. et al. DPPH assay of vegetable oils and model antioxidants in protic and aprotic solvents. Talanta, v. 109, n. 0, p. 13-19, 2013.

RUFINO, M. S. M. et al. Free radical-scavenging behaviour of some north-east Brazilian fruits in a DPPH system. Food Chemistry, v. 114, n. 2, p. 693695, 2009.

RYSZ, J. et al. Serum Antioxidant Capacity is Preserved in Peritoneal Dialysis Contrary to Its Robust Depletion After Hemodialysis and Hemodiafiltration Sessions. Therapeutic Apheresis and Dialysis, v. 14, n. 2, p. 209-217, 2010.

SÁNCHEZ-MORENO, C.; LARRAURI, J. A.; SAURACALIXTO, F. A procedure to measure the antiradical efficiency of polyphenols. Journal of the Science of Food and Agriculture, v. 76, n. 2, p. 270-276, 1998.

SCHERER, R.; GODOY, H. T. Antioxidant activity index (AAI) by the 2,2-diphenyl-1-picrylhydrazyl method. Food Chemistry, v. 112, n. 3, p. 654-658, 2009.

SHARMA, O. P.; BHAT, T. K. DPPH antioxidant assay revisited. Food Chemistry, v. 113, n. 4, p. 1202-1205, 2009.

SOLER-RIVAS, C.; ESPÍN, J. C.; WICHERS, H. J. An easy and fast test to compare total free radical scavenger capacity of foodstuffs. Phytochemical Analysis, v. 11, n. 5, p. 330-338, 2000.

TINKEL, J.; HASSANAIN, H.; KHOURI, S. J. Cardiovascular Antioxidant Therapy: A Review of Supplements, Pharmacotherapies, and Mechanisms. Cardiology in Review, v. 20, n. 2, p. 77-83, 2012.

TSIMOGIANNIS, D. I.; OREOPOULOU, V. Free radical scavenging and antioxidant activity of $5,7,3^{\prime}, 4^{\prime}$-hydroxy-substituted flavonoids. Innovative Food Science \&amp; Emerging Technologies, v. 5, n. 4, p. 523-528, 2004.

VALGIMIGLI, L. et al. Kinetic Solvent Effects on Hydroxylic Hydrogen Atom Abstractions Are Independent of the Nature of the Abstracting Radical. Two Extreme Tests Using Vitamin E and Phenol. Journal of the American Chemical Society, v. 117, n. 40, p. 9966-9971, 1995.

VILLAÑO, D. et al. Comparison of antioxidant activity of wine phenolic compounds and metabolites in vitro. Analytica Chimica Acta, v. 538, n. 1-2, p. 391-398, 2005.

YAO, $\mathrm{H}$. et al. Screening and quantitative analysis of antioxidants in the fruits of Livistona chinensis $\mathrm{R}$. Br using HPLC-DAD-ESI/MS coupled with pre-column DPPH assay. Food Chemistry, v. 135, n. 4, p. 28022807, 2012.

Rev. Bras. PI. Med., Campinas, v.17, n.1, p.45-50, 2015. 